\title{
ETHNICITY AND IMPRESSIONS OF PERSONALITY USING THE FIVE-FACTOR \\ MODEL: STEREOTYPING OR CULTURAL SENSITIVITY?
}

\author{
Andrea Kay Cooper \\ San Juan College \\ David Chin Evans \\ University of Washington
}

This research begins to answer the question: why do we, as humans, consistently draw conclusions about others based on generalized information? If we move away from the assumption that all stereotypes are inaccurate and harmful, then we can begin to understand this behavior. Lee et al. (2013) suggest that people stereotype others and generalize information to all the members of a specific group when there is an absence of "relevant individuating information" (pg. 478). In other words, people make conclusions based on the evidence that is available. Lee et al. describe these generalizations as weak and provide evidence that people reject these stereotypes when better evidence is available. We view this as a refinement of conclusions, rather than weak or inaccurate. Human beings are able to gather the best possible information and evaluate it. When more accurate information is available, then we are able to refine our conclusions. There is little evidence to suggest that people cling to stereotypes without thought or evaluation, and in fact, we readily reject generalizations when more information is available (Lee et al., 2013).

Neuberg and Sng (2013) describe a framework for understanding stereotyping behavior and contribute additional support showing that stereotypes can be utilized as a way to gather useful information about others. The authors approach the discussion with an evolutionary perspective, specifically Life History Theory, and argue that the complexity of our social systems necessitates making generalizations in order to protect one's self or find a mate. Not only is the ability to draw conclusions about those around us important to navigate the social world, but it may actually provide fitness benefits. Those who are also able to refine generalizations when more information is available will be even more successful navigating our complex social systems. 
Social scientists have for some time sensed the need to shift their conception of stereotypes from the perspective of prejudice theory, which focuses on negative predispositions and discrimination toward out-groups, to the perspective of interpersonal perception theory, which focuses on the mutual impressions formed by members of distinct human groups. The 1995 publication of Stereotype Accuracy, an edited volume by Lee, Jussim, and McCauley arising from a conference of the American Psychological Association Science Directorate, serves as well as any other as a signpost for this shift. This volume argued that many of the features that might distinguish stereotypes from group impressions are either logically untenable (e.g. that they are all-or-none beliefs, illogically resistant to contradictions, and factually wrong for reasons of hearsay) or have received little empirical support (e.g. that they lead people to ignore individual differences, stem from negative attitudes, and imply genetic essentialism). Perhaps more importantly, the volume pointed out that two key features of stereotypes as group impressions remain virtually unmeasured: their specific content and their degree of accuracy or falsehood. More recently several researchers have continued investigating the utility of stereotyping behavior and call for researchers to look beyond the traditional view of stereotypes (Koenig \& Early, 2014; Jussim, 2005, 2012; Jussim et al., 2009; Lee et al., 2013).

Given the abundant research on stereotypes in recent years, why is there so little data about what people think of various racial and ethnic groups and whether they are right? Many scholars (Ottati \& Lee, 1995; Stangor \& Schaller, 1996; Zebrowitz, 1996) have noted that even basic research on what personality traits are ascribed to racial groups, which is the focus of the present paper, has progressed little since the 1933 Princeton studies by Katz \& Braly. Scholarly work has instead focused on the formation, maintenance, and activation of stereotypes as cognitive representations, thus more often measuring reaction times, error rates, and variability rather than semantic content or trait-level beliefs. As Ottati and Lee (1995, p. 32) remarked, and others agree (Ryan \& Bogar, 2001; Zebrowitz, 1996), "recent research has focused almost exclusively on the cognitive process of stereotyping. This focus on process, which is by no means without value, has failed to address the question of whether stereotypes possess accurate content in real-world contexts."

Perhaps more fundamentally, because this field began in the sociopolitical context of WWII and the Civil Rights era, "stereotypes" originally referred to absurd propaganda images and Jim Crow portrayals (Lippman, 1965; Fixico, 2011). As such, any serious consideration of their accuracy was antithetical almost by definition and continues to be at odds with the human rights advocacy that motivates much of the scholarly interest in this area. The term "stereotype accuracy" is as uninviting 
for many researchers today as "communist liberty" would have been in the 1950s or "segregationist equality" in the 1960s. Lee, McCauley, \& Jussim (1995) report one incident in which a reviewer of one of their stereotype accuracy manuscripts asked them "What should we be doing? Articles with titles like 'Are Blacks really lazy?' and 'Are Jews really cheap?'?” (p. 310). Cronbach called for more research on the influence of stereotypes on impression accuracy in 1955 and Lee et al. repeated the call in Stereotype Accuracy in 1995. Perhaps to understand why there is still too little data on key questions in this area, we need look no further than the title of their volume.

But by approaching stereotypes as group impressions consistent with interpersonal perception theory (Brunswick, 1956; Cronbach, 1955; Funder, 1999; Kenny, 1994, 2004), and unburdening it of the presumption of prejudice as Lee et al. $(1995,2009,2013)$ recommend, a better alignment is achieved with the current global context of group beliefs in a way that does not impede much needed research. Many theorists have long rejected the view that stereotypes are fixed, negative, all-or-none "pictures in the head" (Lippman, 1965) but are instead probabilistic expectations that people hold about the characteristics of groups, which may be positive or negative, accurate or inaccurate (LeVine \& Campbell, 1972; McCauley \& Stitt, 1978; Jussim 2009). People may utilize visible cues about others' race and ethnicity to form impressions about their personalities (Brunswick, 1956) in the same way they utilize dress, smiling, hairstyle (Zebrowitz \& Collins, 1994) and facial symmetry (Noor \& Evans, 2003).

Specifically, this study provides insight into whether impressions based on ethnicity help or hinder the ability to form impressions that agree with the self-views of the target groups or members, and does group membership have "cue validity?" Cue validity, also called "agreement," may only be determined by comparing beliefs against some criterion, that is, some data about the stereotyped target group. How is this accomplished? First, we must first shift from studying stereotypes of "races" (e.g. Native Americans) to stereotypes of "ethnic groups," (e.g. Native American people on a particular college campus). It is necessary to define the stereotyped target group in a way that represents a real population, rather than an abstract concept, from which a generalizable criterion sample may be drawn. Ethnicity is tightly connected to particular cultural traits that identify a group of people. While biological differences between populations do exist, these differences do not support the existence of distinct racial groups based on any suite of genetic traits and hold little potential for understanding each other. Cultural traits are better able to help us understand each other, so ethnicity is an important cue to 
utilize when forming impressions. Knowing someone's skin color, however, provides little insight into a person's behavior or personality.

Second, we must choose our criterion variable. Since we are studying personality impressions, the options include self-impressions, peerratings, or behavioral personality indicators. Since peer-ratings may themselves be influenced by stereotypes, and behavioral personality indicators are not perfectly established and impractical to gather, we focused on self-impressions. Thus we are not testing the "accuracy" of the stereotypes, but whether "people see others as they see themselves," a nontrivial aspect of intergroup dynamics. If ethnic group impressions show high cue validity, using them may be a form of cultural sensitivity; if however they show low cue validity, using them may lead to stereotypic inaccuracy.

In this article, the term impression will be used (with modifiers) rather than the term stereotype, but in all cases refers to impressions of personality on the Five Factor domains (see Kenny, 1994). A self-impression is a perceiver's impression of his or her own personality, and an other-impression is a perceiver's impression of another individual's personality. A group-impression is a perceiver's impression of an entire group of people, abstract or real, and may be either an in-group-impression if the perceiver is a member of the group or an out-group-impression if the perceiver is not a member of the group. All of the above may be aggregated across theoretically significant groups of perceivers. To this point, aggregated self-impressions of, for example, German or Japanese citizens (Allport, 1954) are quite distinct from in-group-impressions given by German or Japanese citizens, as the former impressions are of individuals (selves) and the latter are of groups (in-groups). Comparing these various impressions provides one means of assessing their accuracy, but we will return to that point later.

\section{INTRODUCTION}

Among an interacting population, we hypothesize that impressions on the Five Factor domains will vary systematically depending on the stated ethnic group membership (H1).

\section{Table 1: Five Factor Characteristics Definitions}

\begin{tabular}{|l|l|}
\hline Neuroticism & $\begin{array}{l}\text { Tendency to experience psychological distress like anxiety or } \\
\text { depression }\end{array}$ \\
\hline Extraversion & $\begin{array}{l}\text { Active, sociability, experience of positive emotions, excitement } \\
\text { seeking }\end{array}$ \\
\hline Open & Open to new experiences, artistic, behaviorally flexible \\
\hline Agreeable & Trusting, sympathetic, cooperative \\
\hline Conscientiousness & Well-organized, structured, compulsive \\
\hline
\end{tabular}


We also hypothesize that group-impressions will be similar to aggregated self-impressions, but group-impressions will be exaggerated across all domains (H2). Lastly, we expect that In-group impressions will agree more with aggregated self-impressions than will Out-group impressions (H3).

\section{PARTICIPANTS}

Students attending a northern New Mexico College participated in the research. The population mainly consists of White, Native American, and Hispanic/Latino residents. The College has a high percentage of Native American students, many of whom are Diné (Navajo) given the close proximity of the Navajo Nation. This population presented a unique research location and provided insight into ethnic impressions in a community that interacts daily. The sample included 477 participants (18 or older) representing the ethnic make-up of the college (Table 2). White and Native American groups are in the majority while Hispanic/ Latino groups remain the minority in the study sample and the population. The sample included 351 (74\%) women and 126 (26\%) men.

\section{TABLE 2: ETHNIC GROUP DEMOGRAPHICS}

\begin{tabular}{|l|c|c|c|}
\hline & $\begin{array}{c}\text { White/European } \\
\text { American }\end{array}$ & Native American & Hispanic/Latino \\
\hline Study Sample & $44 \%$ & $36 \%$ & $14 \%$ \\
\hline $\begin{array}{l}\text { College } \\
\text { Demographics }\end{array}$ & $56 \%$ & $23 \%$ & $13 \%$ \\
\hline
\end{tabular}

Studying ethnicity ultimately allows us to understand people better as individuals rather than products of perceptions formed by others, yet systematic analysis requires asking research participants to put themselves in a distinct ethnic category (i.e. White, Native American, Hispanic/Latino). We know that these categories do not fully represent the diversity among all people. To ask someone to identify herself as Native American does not take into account her particular subculture. For example, Navajo culture differs substantially from Puebloan cultures. However, given the requirements of statistical analysis, ethnicity categories must be created. We did ask respondents to identify their ethnicity as they define it, which produced interesting qualitative data. Many respondents used the same terms used in data analysis, but many of the responses tended to be more specific or far more general than our created categories. For instance, some identified their ethnicity as "Diné," "Seminole/Navajo," and "Anglo Southwestern American." More general responses included, "human," "mix of everything," and "American." Perhaps outside the scope of this paper and not usable in analysis, their 
answers are no less important in understanding how individuals identify themselves.

\section{Methods}

Participants were asked to complete a twenty-one item survey from the Big Five Inventory (BFI-K Form S; John 2005) on a five point scale that was used to calculate their Five-Factor personality traits or self-impression as well as a group-impression of a randomly selected ethnic group. Participants were only presented with one ethnic group to reduce possible bias caused by comparisons between groups.

The survey specifically asked participants to think of groups at the college, providing data about the interactions between real people and ethnic groups rather than in the abstract. Five Factor personality profiles were created for self-impressions of individuals and for impressions of White, Native American, and Hispanic/Latino ethnic groups.

\section{RESULTS}

Self-impressions were broken down by ethnic group, focusing on the numerically largest groups in the population: White/European American, Native American, and Hispanic/Latino groups on campus. The selfimpression curves resemble the norming curve across all ethnicities. Using a Bonferroni corrected critical value of $\mathrm{p}=.002$ (unless otherwise noted), an initial investigation revealed that people assume significantly different self-impressions of personality across ethnicities $(F(8,1772)=$ 2.576, $\mathrm{p}=.009$ ).

ANOVAs (with a Bonferroni corrected critical value of $\mathrm{p}=.002$ ) were then calculated across specific ethnic group combinations and personality domains. Self-impressions of personality were similar across ethnicity for Openness and Neuroticism. A between subjects ANOVA revealed significant differences between Hispanic/Latino and Native American students in Extraversion $(\mathrm{F}=14.121 ; \mathrm{p}=.00)$. Agreeableness was nearly significant between White and Native American respondents $(\mathrm{F}=5.209 ; \mathrm{p}=.02)$. Lastly, Hispanic/Latino and Native American participants varied significantly in Conscientiousness $(\mathrm{F}=5.624 ; \mathrm{p}=.01)$ See Fig. $1 \mathrm{c}$ and Table 3. 
Table 3: Means and Standard Errors (SE)

\begin{tabular}{|c|c|c|c|c|c|c|c|c|c|c|}
\hline \multirow[b]{2}{*}{ Domain } & \multirow[b]{2}{*}{ Target Label } & \multicolumn{3}{|c|}{$\begin{array}{l}\text { Outgroup-Impressions } \\
\text { (Figs. la \& 2a-c) }\end{array}$} & \multicolumn{3}{|c|}{$\begin{array}{l}\text { Ingroup-Impressions } \\
\text { (Figs. 1b \& 2a-c) }\end{array}$} & \multicolumn{3}{|c|}{$\begin{array}{l}\text { Self-Impressions } \\
\text { (Figs. 1c \& 2a-c) }\end{array}$} \\
\hline & & $\mathrm{N}$ & Mean & SE & $\mathrm{N}$ & Mean & SE & $\mathrm{N}$ & Mean & SE \\
\hline \multirow[t]{3}{*}{ Neuroticism } & White/Euro-American & 59 & 3.013 & 0.077 & 52 & 2.942 & 0.104 & 208 & 2.894 & 0.061 \\
\hline & Latino/Hispanic & 109 & 2.823 & 0.057 & 19 & 2.803 & 0.171 & 69 & 2.786 & 0.106 \\
\hline & Native American & 130 & 2.992 & 0.052 & 76 & 3.092 & 0.086 & 169 & 2.857 & 0.067 \\
\hline \multirow[t]{3}{*}{ Extraversion } & White/Euro-American & 59 & 3.564 & 0.088 & 52 & 3.490 & 0.100 & 208 & 3.262 & 0.054 \\
\hline & Latino/Hispanic & 109 & 3.268 & 0.065 & 19 & 3.908 & 0.165 & 69 & 3.500 & 0.094 \\
\hline & Native American & 130 & 2.388 & 0.059 & 76 & 2.760 & 0.082 & 169 & 3.123 & 0.060 \\
\hline \multirow[t]{3}{*}{ Openness } & White/Euro-American & 59 & 3.549 & 0.078 & 52 & 3.527 & 0.099 & 208 & 3.864 & 0.042 \\
\hline & Latino/Hispanic & 109 & 3.328 & 0.058 & 19 & 3.589 & 0.165 & 69 & 3.861 & 0.074 \\
\hline & Native American & 130 & 3.500 & 0.053 & 76 & 3.600 & 0.082 & 169 & 3.775 & 0.047 \\
\hline \multirow[t]{3}{*}{ Agreeableness } & White/Euro-American & 59 & 2.691 & 0.097 & 52 & 2.822 & 0.110 & 208 & 3.392 & 0.058 \\
\hline & Latino/Hispanic & 109 & 2.897 & 0.071 & 19 & 3.145 & 0.182 & 69 & 3.543 & 0.100 \\
\hline & Native American & 130 & 2.608 & 0.065 & 76 & 2.599 & 0.091 & 169 & 3.590 & 0.064 \\
\hline \multirow[t]{3}{*}{ Conscientiousness } & White/Euro-American & 59 & 3.453 & 0.101 & 52 & 3.486 & 0.097 & 208 & 3.941 & 0.047 \\
\hline & Latino/Hispanic & 109 & 3.374 & 0.074 & 19 & 3.895 & 0.161 & 69 & 4.029 & 0.081 \\
\hline & Native American & 130 & 3.008 & 0.068 & 76 & 3.128 & 0.080 & 169 & 3.808 & 0.052 \\
\hline
\end{tabular}




\section{GROUP IMPRESSIONS}

The impressions formed of entire ethnic groups also vary significantly, showing that different ethnic groups are perceived to have different personalities (ANOVA: $F(8,1756)=21.35, p<.001$ ). Viewing combinations of ethnic groups and personality traits show no significant differences in any of the personality domains between White and Hispanic/Latino group impressions. Native American and Hispanic/Latino participants difference significantly across domains (Neuroticism: ANOVA $\mathrm{F}=7.584, \mathrm{p}=.00$; Extraversion: $\mathrm{F}=104.927, \mathrm{p}=.00$; Openness: $\mathrm{F}=5.147, \mathrm{p}=.02$; Agreeableness: $\mathrm{F}=12.154, \mathrm{p}=.00$; Conscientiousness: $\mathrm{F}=22.989, \mathrm{p}=.00)$. White and Native American group impressions differ significantly only in Conscientiousness (ANOVA $\mathrm{F}=19.186, \mathrm{p}=.00$ ) and Extraversion (ANOVA F=147.630, p=00). See Fig. 1a, 1b, and Table 3.

Figure 1. (a) Outgroup-, (B) Ingroup-, and (c) Aggregated SelfIMPRESSIONS OF WhITE/EURO-AMERICAN, LATINO/HISPANIC, AND Native American people at San Juan College.

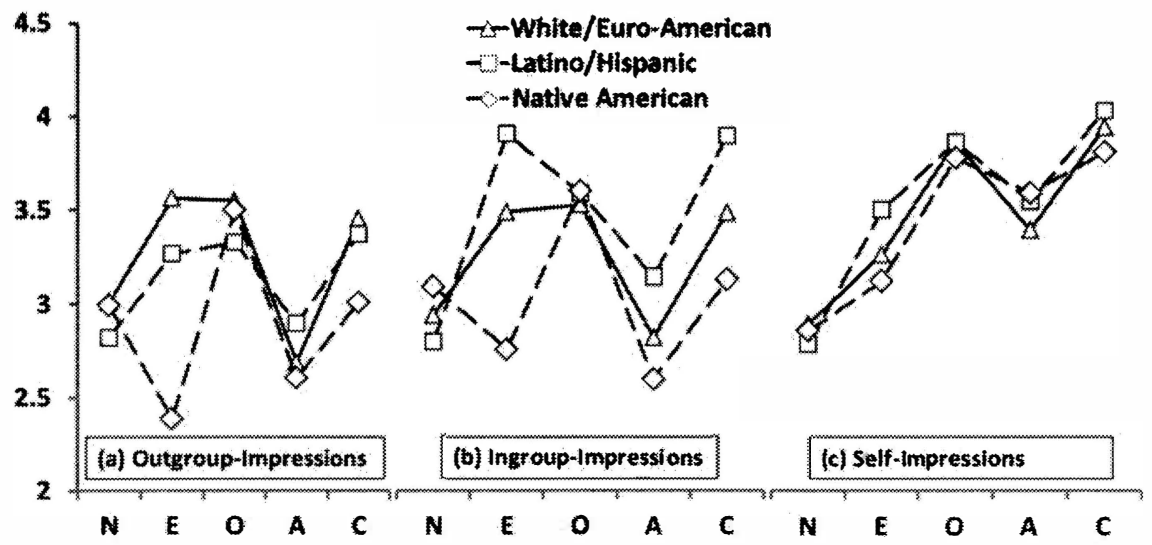

SElF/Group IMPRESSION COMPARISON

Native Americans have significantly lower means for Conscientiousness $(\mathrm{p}=.00)$ and Extraversion $(\mathrm{p}=.00)$ in both the self-impression and the group-impression of Native Americans. This suggests some agreement between how Native Americans see themselves and how others see Native Americans as a group. Similarly, White and Hispanic/ Latino respondents were rated as higher in Conscientiousness and Extraversion. Group-impressions of Conscientiousness and Extraversion are notably lower than self-ratings, but are both in a similar direction.

Ratings of Agreeableness showed considerable disagreement between self-impressions and impressions formed by others. Self-impressions across all ethnicities show high Agreeableness, yet all groups rate 
other groups as low in Agreeableness. More generally, self-impressions are much higher than the impressions formed of ethnic groups across all domains. Despite this difference, the general trend of significant differences across personality traits is similar in the aggregated self- and group-impressions.

\section{IN-GROUP/OUT-GROUP IMPRESSIONS}

Given the study design, individuals of a particular ethnicity were periodically asked to form an impression of their own ethnic group. This created an In-group impression. Out-group impressions were formed by those of a different ethnicity than their own. Impressions of the In-group are expected to agree more with self-impressions. The initial analysis of the interaction between ethnicity, in/out group, and personality domain showed no significant differences (ANOVA: $F(8,1756)=1.468, p=.164$ ).

Despite no initial findings, the sample was analyzed by each ethnic group. 52 White participants provided personality impressions of Whites as a group, while $110 \mathrm{Hispanic/Latino}$ and Native American participants provided the Out-group impressions for White students. The In-group and Out-group impressions were quite similar and show no significant differences (ANOVA: $F(4,436)=.399, p=.810$ ). See Figure $2 b$ and Table 3.

Figure 2. Outgroup-, Ingroup-, and Aggregated SelfImpressions of (A) Native American (B) White/Euro-American, and (C) Latino/Hispanic people at San Juan College.

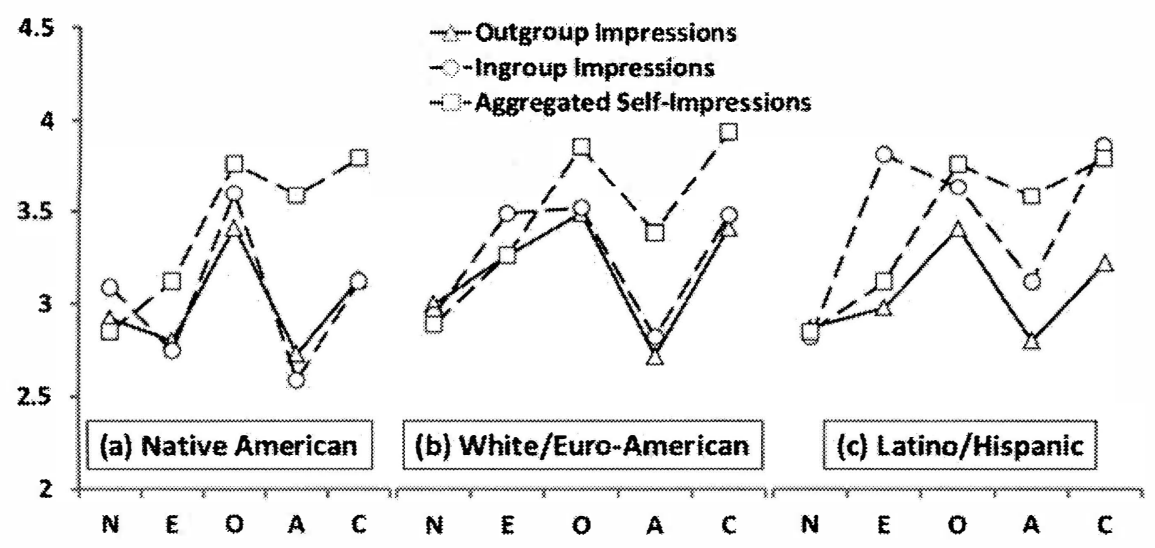

Seventy-six Native American respondents provided personality impressions of Native American students, and 243 Hispanic/Latino and White students rated Native American students as the Out-group. Similar to the White results, In-group and Out-group impressions of Native American 
students are very similar and show no significant differences in value or pattern (ANOVA: $F(4,816)=1.993, \mathrm{p}=.094)$ See Figure $2 \mathrm{a}$ and Table 3.

Nineteen Hispanic/Latino participants provided In-group impressions, and 263 participants provided Out-group impressions of Hispanic/ Latino students. The curves contrast with the trends seen among White and Native American students and indicate disagreement across all domains (ANOVA: $F(4,504)=3.046, p=.017)$. Specifically, In and Out group impressions of Hispanic/Latinos revealed significant differences in Conscientiousness (ANOVA: F $(1.408)=10.768, \mathrm{p}=.001$ ) and Extraversion $($ ANOV A: $F(1.425)=15.589, p=.000)($ See Fig. $2 \mathrm{c}$ and Table 3$)$

\section{CONCLUSION}

In this population, aggregated self-impressions show agreement with the impressions that others hold in traits, Conscientiousness and Extraversion. Disagreement exists in Agreeableness between self-impressions and the impressions of others. Lastly, in-group and out-group ratings showed similar patterns (although not significant) for the two majority groups (White and Native American students) while showed a dissimilar pattern for the minority group (Hispanic/Latino students). These results indicate that cultural characteristics of Native Americans and Whites in this population are conveyed in a manner that allows crosscultural understanding. Hispanic/Latino students do not appear to be seen as they see themselves in this population. The low sample size must be noted, which can make interpretation more difficult.

This research expands the study of stereotyping by establishing that people use ethnicity as a cue to understand others and that the use of ethnicity sometimes demonstrates cultural sensitivity rather than stereotypes based on inaccurate perceptions. The present study also explores stereotyping behavior in a population with a large Native American population. As Donald L. Fixico acknowledges, many people do not have much knowledge of Native American groups and he calls for more research and exposure (2013). He proposes exactly what our study demonstrates, namely that once interacting populations have enough contact with each other, they are likely to be understood by each other.

\section{Discussion}

Our data suggest that individuals do utilize stereotypes and create exemplars of an entire culture as a way of navigating a complex social world. These exemplars serve to help predict social interactions with people whom we do not know. Cultural exemplars will hold varying degrees of accuracy but are created in the human mind as a strategy to help categorize and organize interpersonal interactions. 
We might consider the use of ethnicity as a cue to personality reflective of real, observable cultural differences. Cultural differences certainly exist and many conflicts between groups of people are based on cultural misunderstandings. If ethnicity is a cultural construct, as we know it is, and then it follows that ethnicity is a good clue to use when trying to understand and interact with the people around us. This research presents evidence that the use of ethnicity to form impressions of others is based on cultural awareness and sensitivity rather than misunderstanding and bias. Impressions of others seem to be moving beyond inaccurate prejudice to a more useful form of cultural awareness. Of course, impressions of an entire group of people based on only a few characteristics, ethnicity or otherwise, will be generalized and fail to capture individuals completely. We argue that ethnicity can be used as a valid predictor of personality if we are aware of each other and our varying cultures.

Our results could also indicate that societal roles do exist in terms of minority and majority group status. Minority groups are bound to be less understood than majority groups due to the presence of bias but also because majority culture will be prevalent in all realms of society drowning out much of minority culture. Our results indicate that minority and majority groups can achieve a level of understanding and cultural awareness. Hispanic/Latino groups, the numerical minority in this population, seem to be misunderstood and reveal that personality impressions can be misleading and inaccurate when understanding is lacking.

\section{LIMITATIONS}

All data is self-reported and given the sensitivity of discussions of ethnicity, respondents may be inclined to answer less truthfully. Observational and other forms of data would supplement this study and should be considered for future research in this area. The data also aggregate individuals based on ethnicity and do not allow for subcultural or individual differences. Future research should also include dyadic encounters between individuals to further understand how we use ethnicity to understand others on all levels of analyses. We hope this research spurs more research using the Five Factor model of personality.

\section{REFERENCES}

Allport, G.W. (1954). The nature of prejudice. Cambridge, MA: Addison-Wesley.

Brunswik, E. (1956). Perception and the representative design of psychological experiments. Berkeley: University of California Press. 
Cronbach, J.L. (1955). Processes affecting scores on "understanding of others" and "assumed similarity." Psychological Bulletin, 52, 177193.

Fixico, Donald L., (2000). The Urban Indian Experience in America. Albuquerque: University of New Mexico Press.

Funder, D.C. (1999). Personality judgment : A realistic approach to person perception. San Diego, CA: Academic.

Jussim, L. (2005). Accuracy in social perception: criticisms, controversies, criteria, components, and cognitive processes. In M.P. Zanna (Ed.), Advances in experimental social psychology. 37:1-93.

(2012.) Social perception and social reality: why accuracy dominates bias and self-fulfilling prophecy. New York: Oxford University Press.

Jussim, L. et al. (2009). The unbearable accuracy of stereotypes. In T. Nelson (Ed.), The handbook of prejudice, stereotyping, and discrimination. Mahwah, NJ: Erlbaum.

Katz, D., \& Braly, K. (1933). Racial prejudice and racial stereotypes. Journal of Abnormal and Social Psychology, 30, 175-193.

Kenny, D.A. (1994). Interpersonal perception: A social relations analysis. New York, NY: Guilford.

Kenny, D.A. (2004). A general model of interpersonal perception. Personality and Social Psychology Review, 8, 265-280.

Koenig, A. \& Early, A. (2014). Evidence for the social role theory of stereotype content: observations of groups' roles shape stereotypes. Journal of Personality and Social Psychology. 107(3):371-392.

Lee, Y.-T, Jussim, L.J. . \& McCauley, C.R., (1995). Stereotype accuracy: Toward appreciating group differences. Washington, DC: American Psychological Association.

(2013). Stereotypes as valid categories of knowledge and human perceptions of group differences. Social and Personality Psychology Compass. 7(7): 470-486.

LeVine, R. A., \& Campbell, D. T. (1972). Ethnocentrism: Theories of conflict, ethnic attitudes, and group behavior. Oxford, UK: Wiley.

Lippman, W. (1965). Public opinion. New York, NY: Free Press.

McCauley, C., \& Stitt, C.L. (1978). An individual and quantitative measure of stereotypes. Journal of Personality and Social Psychology, 36, 929-940. 
Noor, F., \& Evans, D.C. (2003). The effect of facial symmetry on perceptions of personality and attractiveness. Journal of Research in Personality, 37, 339-347.

Neuberg, S. \& Sng, O. (2013). A life history theory of social perception: stereotyping at the intersections of age, sex, and ecology (and race). Social Cognition, 31(6), 696-711.

Ottati, V., \& Lee, Y.T. (1995). Accuracy: A neglected component of stereotype research. In L.J. Jussim, C.R. McCauley, \& Y.T. Lee (Eds.) Stereotype accuracy: Toward appreciating group differences (pp. 29-59). Washington, DC: American Psychological Association.

Ryan, C.S., \& Bogart, L.M. (2001). Longitudinal changes in the accuracy of new group members' in-group and out-group stereotypes. Journal of Experimental Social Psychology, 37, 118-133.

Ryan, C.S. (2002). Stereotype accuracy. In W. Stroebe \& M. Hewstone (Eds.), European review of social psychology. (13): 75-109. Hove, England: Psychology Press/Taylor \& Francis.

Stangor, C., \& Schaller, M. (1996). Stereotypes as individual and collective representations. In C.N. Macrae, C. Stangor, \& M. Hewstone (Eds.), Stereotypes and stereotyping (pp. 3-37). New York, NY: Guilford Press.

Zebrowitz, L.A. (1996). Physical appearance as a basis of stereotyping. In C. N. Macrae, C. Stangor, \& M. Hewstone (Eds.), Stereotypes and stereotyping (pp. 79-120). New York, NY: Guilford Press.

Zebrowitz, L. A., \& Collins, M. A. (1997). Accurate social perception at zero acquaintance: The affordances of a Gibsonian approach. Personality and Social Psychology Review, 1, 203-222. 